Supplemental information

\title{
Organic Photodiode Integration on Si Substrate Beyond 1000 nm Wavelength
}

Lai-Hung Lai ${ }^{*}$, Chin-Chuan Hsieh ${ }^{1}$, Jhao-Lin $\mathrm{Wu}^{2}$, Yi-Ming Chang ${ }^{2}$

${ }^{1}$ VisEra Technologies Company Limited, No. 12, Dusing Rd. 1Hsinchu Science Park, Hsinchu City 30078, Taiwan

${ }^{2}$ RaynergyTek Inc., 2F, No. 60, Park Avenue 2, Hsinchu Science Park, Hsinchu 30844, Taiwan

* Corresponding author:

E-mail: Larry_Lai@viseratech.com

Keywords: Infrared organic photodiode, CMOS image sensor, organic photodetector, organic photoactive layer, bottom electrode

\section{The device architecture for OPD and CMOS ROIC integration}

The photodiode structure is illustrated in Figure S1a, where p-Si substrate is used, followed by $200 \mathrm{~nm}$ thermal oxide and 100-200 $\mathrm{nm}$ metal. The organic bulk heterojunction photoactive layer with a thickness of $\sim 500 \mathrm{~nm}$ is sandwiched between 
the electron transporting layer $(\mathrm{AZO})$ and the hole transporting layer $(\mathrm{NiO})$. The IZO as a transparent conductive oxide is used as a top electrode. In the organic image sensor (Figure S1b and S1c), each pixel has a photodiode with a bottom electrode connecting to a source and a gate of Si transistors that link to column/row decoder and multiplexer circuits. In this 1D3T (one photodiode and three transistors) scheme, the photodiode is initially reset by $\mathrm{S}_{\mathrm{RST}}$ to a voltage of $\mathrm{V}_{\mathrm{RST}}$. The pixel parasite capacitance of organic bulk heterojunction can be calculated by $C=\kappa \epsilon_{0} A / t$, where the $\kappa$ is dielectric constant, for the organic layer is $\kappa \sim 4, \epsilon_{0}$ is vacuum permittivity $\left(\epsilon_{0}=8.85 \mathrm{E}-12 \mathrm{~F} / \mathrm{m}\right), \mathrm{A}$ is the area of the bottom electrode connecting to the drain of transistors, and $\mathrm{t}$ is the thickness of organic layer between two electrodes. For example, for $10 \mu \mathrm{m}$ pixel size with an organic layer thickness of $500 \mathrm{~nm}$, the capacitance is $7 \times 10^{-15} \mathrm{~F}$. Suppose the $\mathrm{V}_{\mathrm{RST}}$ is $-4 \mathrm{~V}$, the total charge in the photodiode after reset is defined as full well capacity, $\mathrm{Q}=\mathrm{CV}=2.8 \times 10^{-14} \mathrm{C}$, which is equivalent to 177,000 electrons. During the integration period, the voltage of the parasite capacitor drops depending on the light intensity shinning on each pixel. The voltage is amplified and read out by source follower amplifier $\left(\mathrm{S}_{\mathrm{SF}}\right)$ and row selector $\left(\mathrm{S}_{\mathrm{RS}}\right)$ transistors, respectively. 

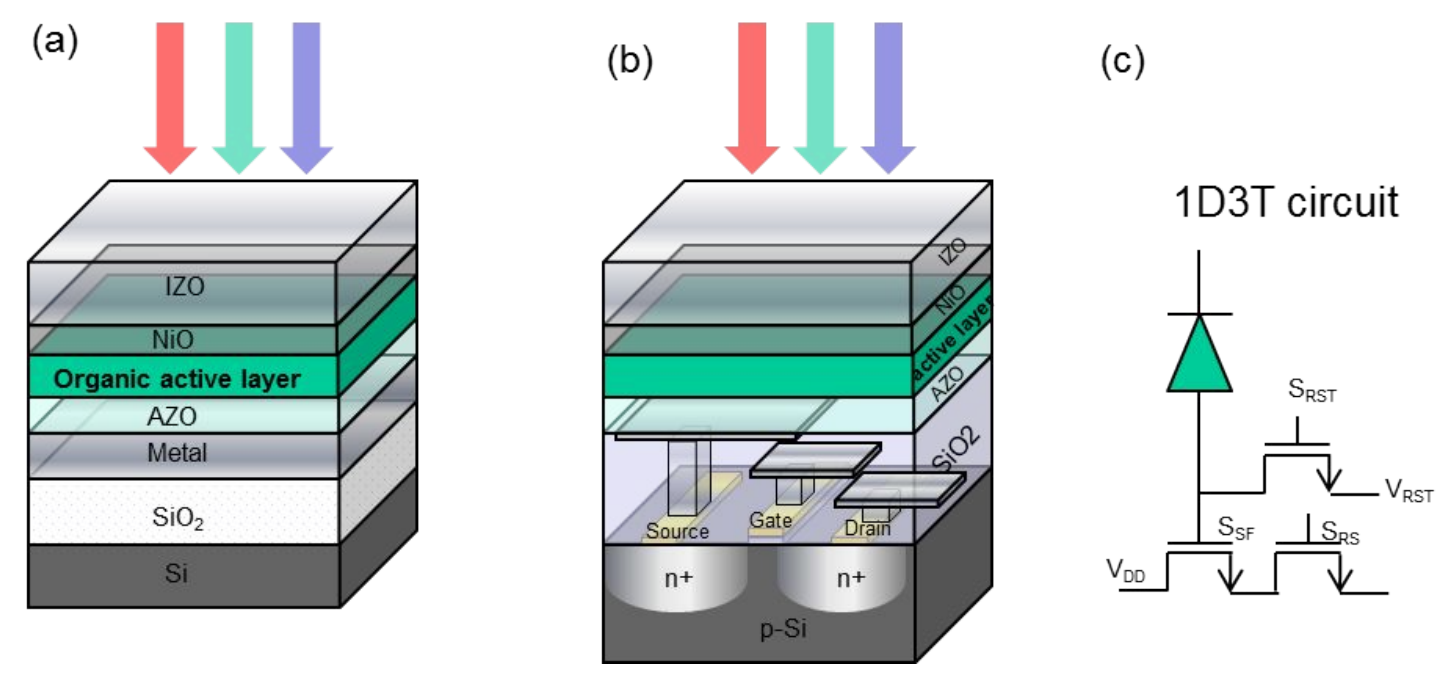

Figure S1. Schematic drawing of (a) organic photodiode integrated on Si substrate,

(b) the scheme of organic CMOS image sensor with bottom electrode connecting to the source of the transistor. (c) The pixel circuit with 1D3T framework.

2. AFM images of the surface topographies and the root mean square roughness of the bottom electrode
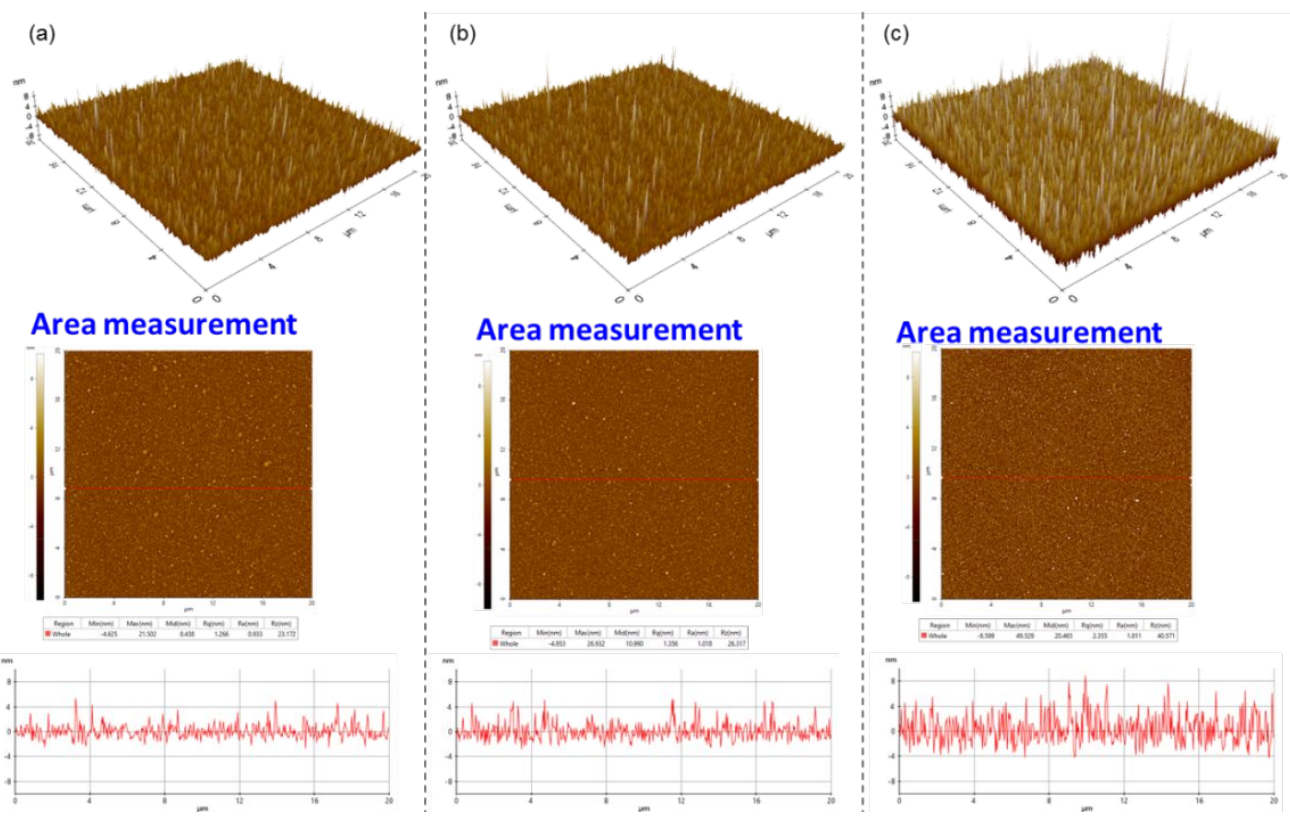
Figure S2. AFM images of the surface topographies of the bottom electrode (a)Al, (b) TiN, (c) W.

Table S1. Surface roughness of different metal surface.

\begin{tabular}{|c|c|c|c|}
\hline unit : nm & $\mathrm{Rq}$ & $\mathrm{Ra}$ & $\mathrm{Rz}$ \\
\hline $\mathrm{Al}$ & 1.495 & 0.934 & 58.996 \\
\hline $\mathrm{Al}$ & 1.266 & 0.933 & 23.172 \\
\hline $\mathrm{Al}$ & 1.468 & 1.058 & 28.451 \\
\hline $\mathrm{Al} / \mathrm{TiN}$ & 2.292 & 1.787 & 35.495 \\
\hline $\mathrm{Al} / \mathrm{TiN}$ & 2.355 & 1.811 & 40.571 \\
\hline $\mathrm{Al} / \mathrm{TiN}$ & 2.290 & 1.765 & 30.504 \\
\hline $\mathrm{Al} / \mathrm{W}$ & 1.356 & 1.018 & 26.317 \\
\hline $\mathrm{Al} / \mathrm{W}$ & 1.606 & 1.104 & 49.433 \\
\hline $\mathrm{Al} / \mathrm{W}$ & 1.603 & 1.106 & 38.744 \\
\hline
\end{tabular}




\section{Materials characterization of $\mathrm{Al}$ on $\mathrm{Si} / \mathrm{SiO}_{2}$ substrate}

The TEM image shows $\mathrm{AlO}_{\mathrm{x}}$ with a thickness of $\sim 15 \mathrm{~nm}$ is formed on the surface of aluminum (Figure S3a, S3b).

(a)

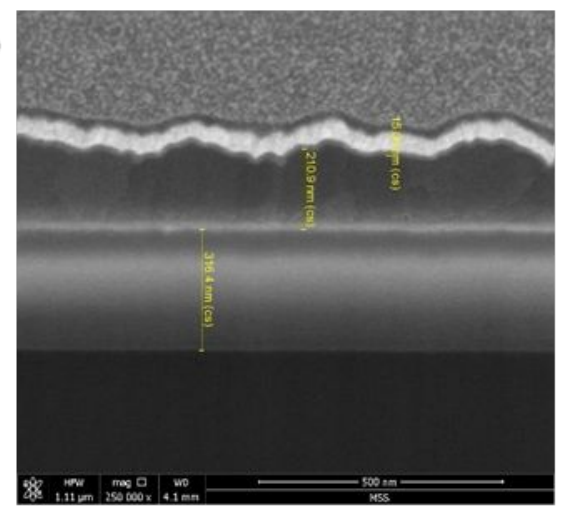

(b)

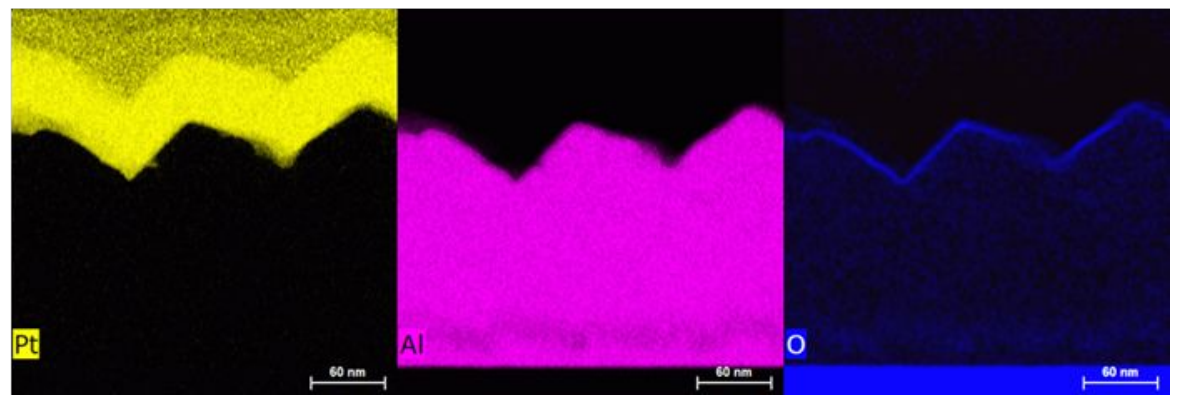

Figure S3. (a) The TEM cross-section image of $\mathrm{Al}$ on $\mathrm{Si} / \mathrm{SiO}_{2}$ substrate. (b) Energy dispersive spectroscopy mapping for materials analysis. 
4. Materials characterization of $\mathrm{W}$ and $\mathrm{TiN}$ on $\mathrm{Si} / \mathrm{SiO}_{2} / \mathrm{Al}$ substrate

(a)

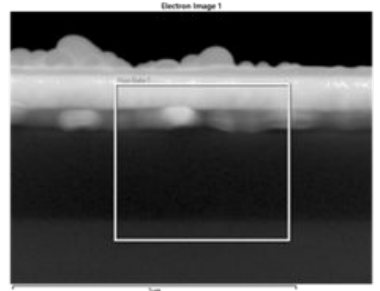

(b)

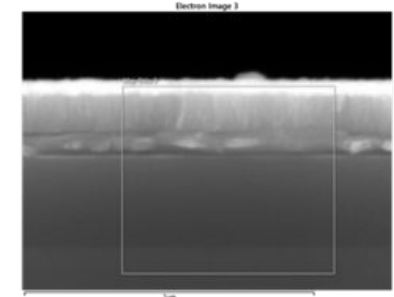

Ti K series

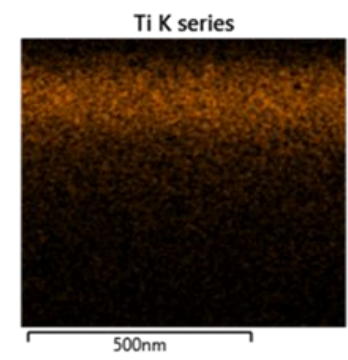

Si K series

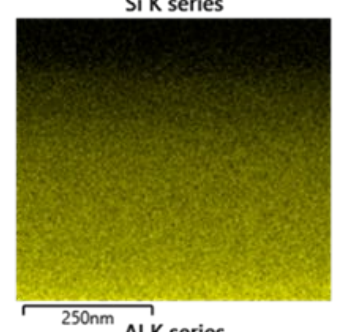

${ }^{250 \mathrm{~nm}}$ Al K series

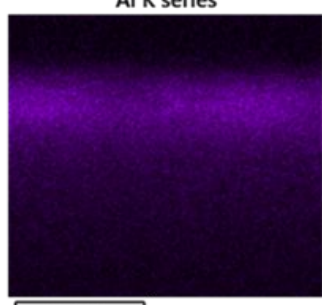

${ }^{250 \mathrm{~nm}} \mathrm{~W} \mathrm{M}$ series

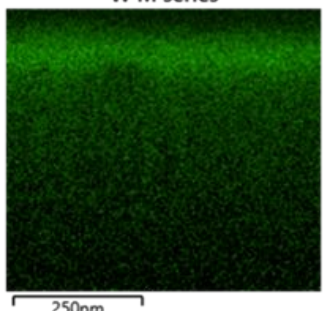

Si K series

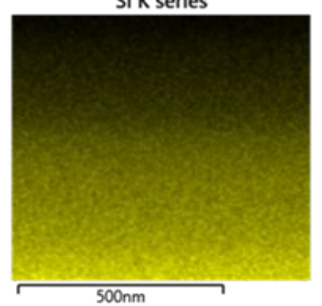

Al K series

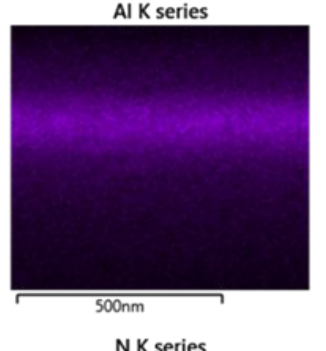

N K series

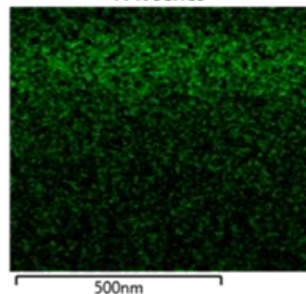

Pt M series

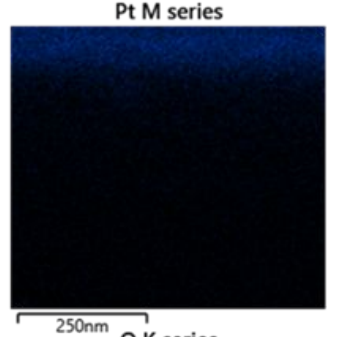

O K series

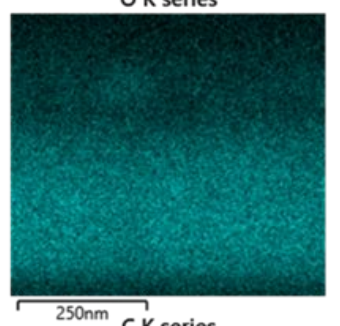

C K series

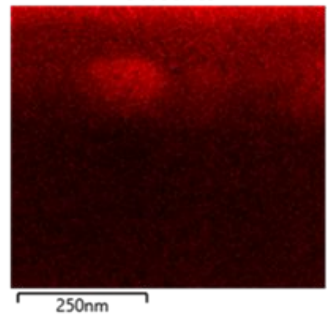

Pt M series

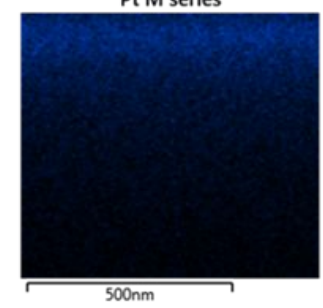

O K series

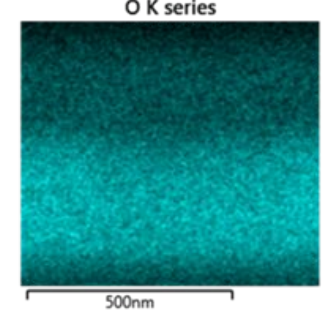

C K series

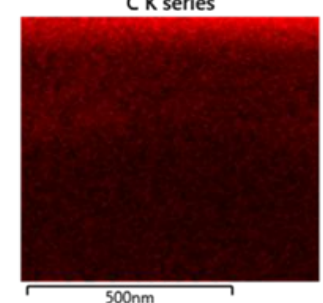

Figure S4. EDS element mapping for (a) W and (b) TiN bottom electrode. 


\section{Dark IV curves fitting}

$I=I_{L}-I_{0}\left(\exp \left(\frac{V+I R_{s}}{n V_{t h}}\right)-1\right)-\frac{V+I R_{s}}{R_{s h}}$

where $\mathrm{I}_{\mathrm{L}}$ is the photocurrent, $\mathrm{I}_{0}$ is the dark saturation current, $\mathrm{V}$ is the applied voltage across the terminals of the diode, $\mathrm{I}$ is the dark current, $\mathrm{V}_{\text {th }}$ is the thermal voltage $\left(\mathrm{V}_{\mathrm{th}}=\mathrm{kT} / \mathrm{q}=26 \mathrm{mV}\right.$ at room temperature $), \mathrm{R}_{\mathrm{s}}$ is series resistance, and $\mathrm{R}_{\mathrm{sh}}$ is shunt resistance, and $\mathrm{n}$ is the ideality factor. The simulated $\mathrm{R}_{\mathrm{s}}, \mathrm{R}_{\mathrm{sh}}$, and $\mathrm{n}$ are shown in Table. 1 .

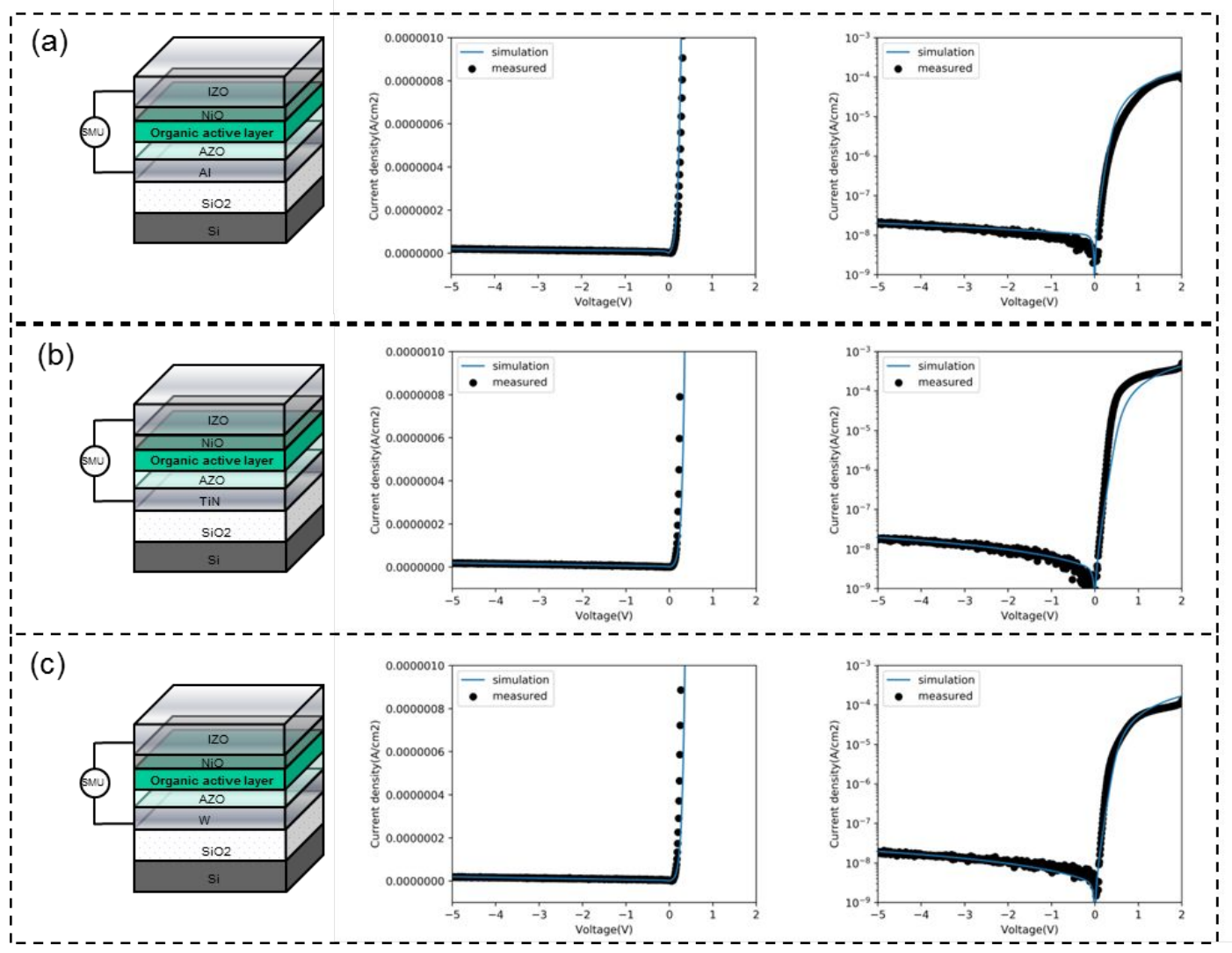

Figure S5. Measured and fitted IV curves of OPD with (a) Al, (b) TiN, and (c) W bottom electrode. 


\section{Error bar of EQE measurement}

At least 8 samples were tested in each condition. The error bar shows the maximum and minimum values of the measurement.
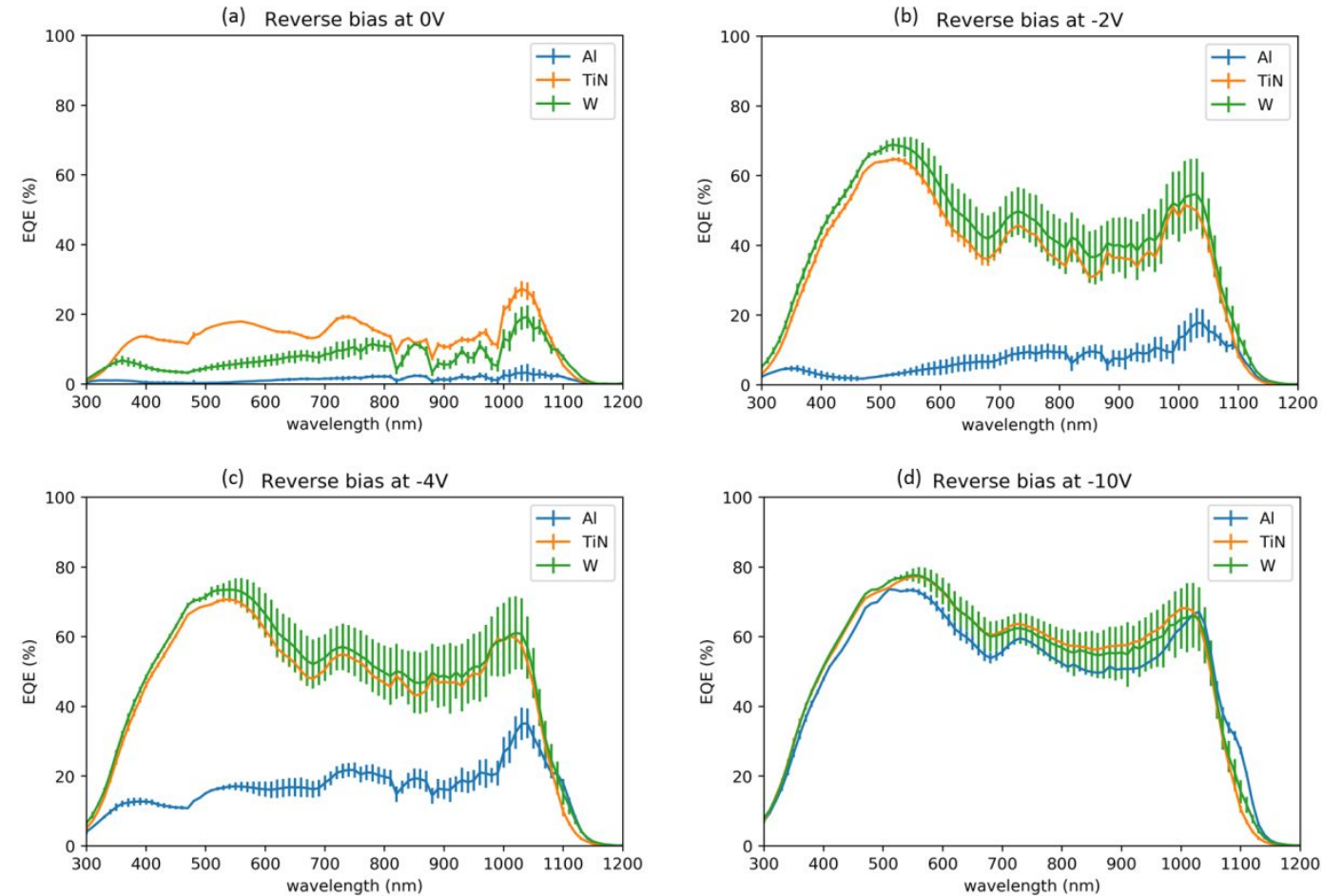

Figure S6. The EQE value and the corresponding error bar at reverse bias (a) 0V, (b) $-2 \mathrm{~V},(\mathrm{c})-4 \mathrm{~V}$, and (d) $-10 \mathrm{~V}$.

7. Absorption spectra of donor and acceptor thin film and the extinction coefficient of the photoactive layer 
(a)

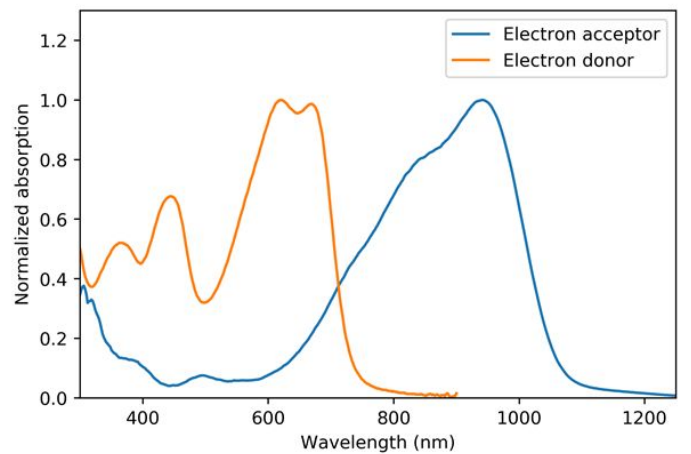

(b)

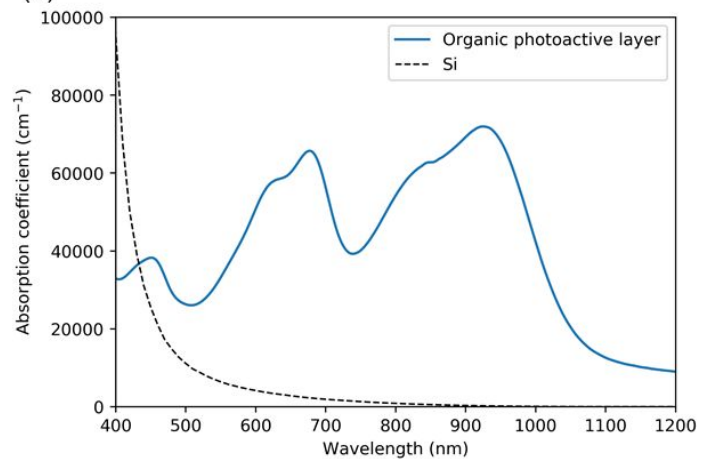

Figure S7. (a) The normalized absorption spectra of acceptor and donor thin film.

(b) the absorption coefficient of the photoactive layer composed by a mixture of donor and acceptor. The black dash line shows the absorption coefficient of $\mathrm{Si}$ as a comparison.

\section{The optical simulation of the organic photodiode on various bottom electrodes}

The optical simulation was done in python by transfer matrix method. The optical constant of each layer used in the simulation is obtained from the web database or from the transmission/reflection spectrum fitting. The optical constants of $\mathrm{Al}, \mathrm{TiN}$ and W are shown in Figure S5. The simulated photon absorption by each layer is shown in Figure S6 and S7. Although the optical constant of bottom electrodes vary a lot, the simulated structure shows that more than $80 \%$ photons are absorbed by the organic active layer with a $500 \mathrm{~nm}$ thickness (Figure S6). While with a $50 \mathrm{~nm}$ thickness of 
organic active layer (Figure S7), the amount of photon absorbed in the organic active layer is affected by the bottom electrodes ascribed to the optical constant of the bottom electrodes.

(a)

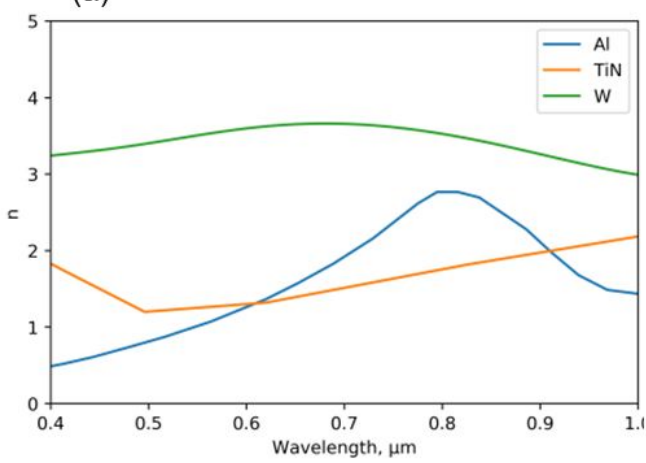

(b)

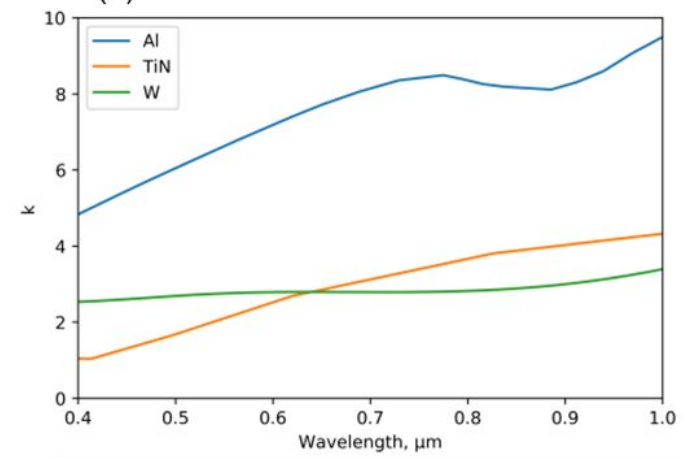

Figure S8. (a) the extinction coefficient and (b) refractive index of metals.

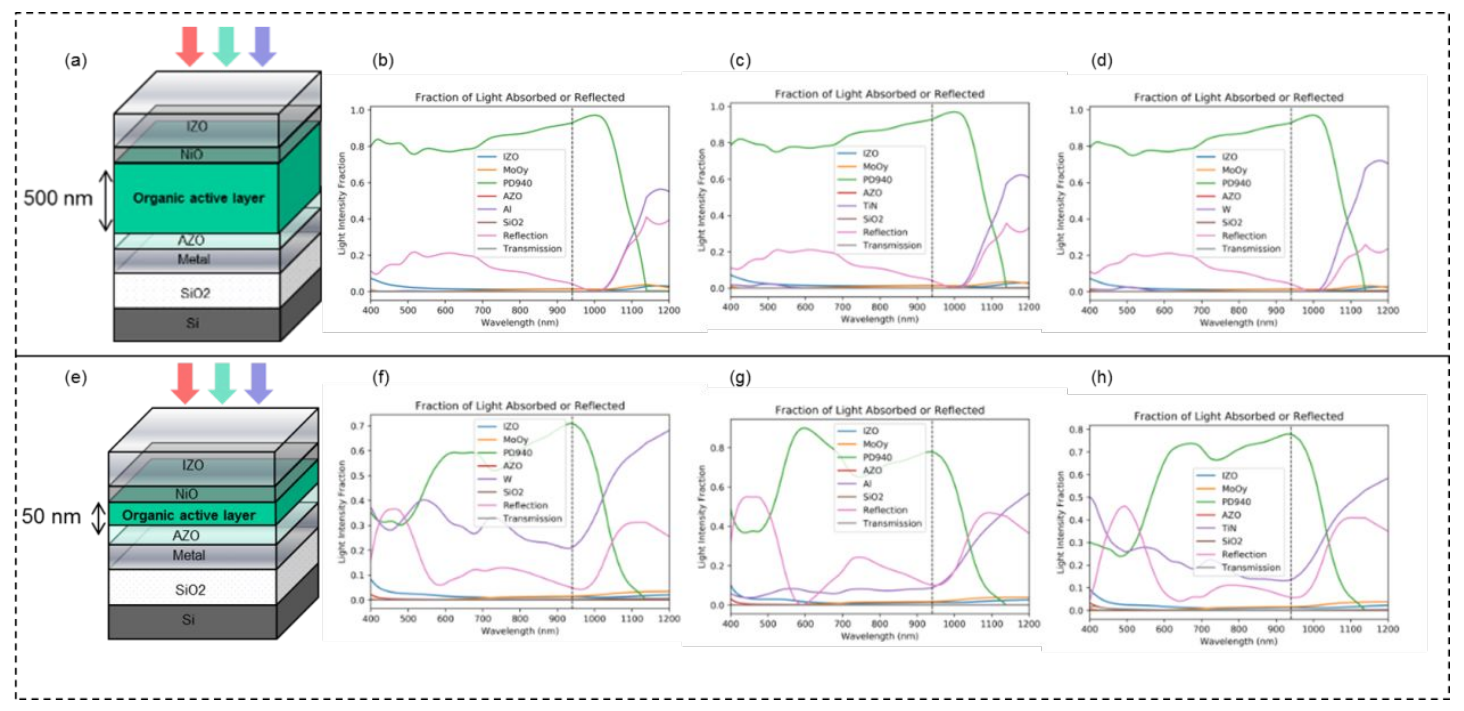

Figure S9. (a,e) The schematic drawing of the device structure, where the organic active layer is $500 \mathrm{~nm}$ and $50 \mathrm{~nm}$, respectively. The simulated light absorption and 
reflection on each layer of organic photodiodes with (b,f) Al, (c,g) TiN, and (d,h) W as different bottom electrodes.

\section{Summary of organic photodiodes reported in the literature}

Table S1 Summary of organic photodiodes reported in the literature.

\begin{tabular}{|c|c|c|c|c|c|c|}
\hline $\begin{array}{l}\text { Processing } \\
\text { solvent a }\end{array}$ & $\begin{array}{l}\text { Device } \\
\text { Structure }\end{array}$ & $\underset{\text { dark }}{\text { I }}\left(A / \mathrm{cm}^{2}\right)$ & EQE (\%) & Detectivity (Jones) & $\underbrace{}_{-3 \mathrm{~dB}}(\mathrm{~Hz})$ & Ref. \\
\hline Halogen-free & $\begin{array}{l}\mathrm{Si} / \mathrm{SiO}_{2} / \mathrm{TiN} / \mathrm{AZO} / \mathrm{ATL} / \mathrm{NiO} / \mathrm{IZO} \\
\mathrm{Si} / \mathrm{SiO}_{2} / \mathrm{W} / \mathrm{AZO} / \mathrm{ATL} / \mathrm{NiO} / \mathrm{ZZO}\end{array}$ & $\begin{array}{l}15 \times 10^{-9} \\
(@-2 \mathrm{~V})\end{array}$ & $\begin{array}{l}\sim 50 \% \text { (@ } 940 \mathrm{~nm} \text { ) 70\% (@ } \\
1030 \mathrm{~nm} \text { ),-4V }\end{array}$ & $\begin{array}{l}2.74 \times 10^{12}(@ 940 \mathrm{~nm} \\
,-4 \mathrm{~V})\end{array}$ & $\begin{array}{l}15 \mathrm{k} \\
(@-4 \mathrm{~V})\end{array}$ & This work \\
\hline Chlorinated & ITO/ZnO/EG40/ATL/MoO $/ \mathrm{Au}$ & $\begin{array}{l}2 \times 10^{-9} \\
(@-2 \mathrm{~V})\end{array}$ & $\begin{array}{l}69.7 \\
\text { (@ } 700 \mathrm{~nm},-2 \mathrm{~V})\end{array}$ & $\begin{array}{l}2.1 \times 10^{13} \\
(@ 700 \mathrm{~nm},-2 \mathrm{~V})\end{array}$ & $\begin{array}{l}72 \mathrm{k} \\
(@-2 \mathrm{~V})\end{array}$ & 1 \\
\hline Chlorinated & ITO/AZO/ATL/PEDOT:PSS/Ag & $\begin{array}{l}\sim 1 \times 10^{-6} \\
(@-5 \mathrm{~V})\end{array}$ & $\begin{array}{l}35.7 \\
\text { (@ } 650 \mathrm{~nm},-5 \mathrm{~V})\end{array}$ & $\begin{array}{l}5.1 \times 10^{11} \\
(@ 630 \mathrm{~nm},-5 \mathrm{~V})\end{array}$ & $\begin{array}{l}222 \mathrm{k} \\
(@-5 \mathrm{~V})\end{array}$ & 2 \\
\hline Chlorinated & ITO/TFB/ATL/Al & $\begin{array}{l}3.4 \times 10^{-11} \\
(@-5 \mathrm{~V})\end{array}$ & $\begin{array}{l}82.0 \\
(@ 660 \mathrm{~nm},-5 \mathrm{~V})\end{array}$ & $\begin{array}{l}2.19 \times 10^{13} \\
(@ 660 \mathrm{~nm},-5 \mathrm{~V})\end{array}$ & $\begin{array}{l}50 \mathrm{k} \\
(@-5 \mathrm{~V})\end{array}$ & 3 \\
\hline Chlorinated & $\mathrm{ITO} / \mathrm{ZnO} / \mathrm{ATL} / \mathrm{MoO}_{3} / \mathrm{Al}$ & $\begin{array}{l}2.1 \times 10^{-8} \\
(@-5 \mathrm{~V})\end{array}$ & $\begin{array}{l}62.4 \\
\text { (@ } 755 \mathrm{~nm},-5 \mathrm{~V})\end{array}$ & $\begin{array}{l}4.57 \times 10^{12} \\
(@ 755 \mathrm{~nm},-5 \mathrm{~V})\end{array}$ & $\begin{array}{l}12.4 \mathrm{k} \\
(@-5 \mathrm{~V})\end{array}$ & 4 \\
\hline Chlorinated & ITO/TIPS pentacene/ATL/Al & $\begin{array}{l}4.0 \times 10^{-9} \\
(@-5 \mathrm{~V})\end{array}$ & $\begin{array}{l}48.1 \\
\text { (@ } 610 \mathrm{~nm},-5 \mathrm{~V})\end{array}$ & $\begin{array}{l}6.61 \times 10^{12} \\
(@ 532 \mathrm{~nm},-5 \mathrm{~V})\end{array}$ & $\begin{array}{l}200 \mathrm{k} \\
(@-5 \mathrm{~V})\end{array}$ & 5 \\
\hline
\end{tabular}




\begin{tabular}{|c|c|c|c|c|c|c|}
\hline-- & ITO/TIPS pentacene/ATL/AI & $\begin{array}{l}9.0 \times 10^{-10} \\
(@-5 \mathrm{~V})\end{array}$ & $\begin{array}{l}70.0 \\
(@ 600 \mathrm{~nm},-5 \mathrm{~V})\end{array}$ & $\begin{array}{l}1.63 \times 10^{13} \\
(@ 530 \mathrm{~nm},-5 \mathrm{~V})\end{array}$ & $\begin{array}{l}10 \mathrm{k} \\
(@-5 \mathrm{~V})\end{array}$ & 6 \\
\hline Chlorinated & ITO/TFB/ATL/Al & $\begin{array}{l}2.0 \times 10^{-8} \\
(@-5 \mathrm{~V})\end{array}$ & $\begin{array}{l}35.0 \\
(\text { @ } 900 \mathrm{~nm},-5 \mathrm{~V})\end{array}$ & $\begin{array}{l}3.34 \times 10^{12} \\
(\text { @ } 900 \mathrm{~nm},-5 \mathrm{~V} \text { ) }\end{array}$ & $\begin{array}{l}63.8 \mathrm{k} \\
(@-5 \mathrm{~V})\end{array}$ & 7 \\
\hline Chlorinated & $\mathrm{ITO} / \mathrm{ZnO} / \mathrm{ATL} / \mathrm{MoO}_{3} / \mathrm{Ag}$ & $\begin{array}{l}3.5 \times 10^{-10} \\
(@-0.1 \mathrm{~V})\end{array}$ & $\begin{array}{l}72.0 \\
\text { (@ } 930 \mathrm{~nm},-0.1 \mathrm{~V} \text { ) }\end{array}$ & $\begin{array}{l}5.1 \times 10^{13} \\
(@ 930 \mathrm{~nm},-0.1 \mathrm{~V})\end{array}$ & $\begin{array}{l}4.5 \mathrm{k} \\
\text { (@ - }-0.1 \mathrm{~V} \text { ) }\end{array}$ & 8 \\
\hline Halogen-free & ITO/PEIE/ATL/MoO $3 / \mathrm{Ag}$ & $\begin{array}{l}1.1 \times 10^{-7} \\
(@-1 \mathrm{~V})\end{array}$ & $\begin{array}{l}38.0 \\
\text { (@ } 680 \mathrm{~nm},-1 \mathrm{~V} \text { ) }\end{array}$ & $\begin{array}{l}1.12 \times 10^{12} \\
(@ 680 \mathrm{~nm},-1 \mathrm{~V})\end{array}$ & $\begin{array}{l}1.02 \mathrm{M} \\
(@-1 \mathrm{~V})\end{array}$ & 9 \\
\hline Chlorinated & $\mathrm{ITO} / \mathrm{ZnO} / \mathrm{ATL} / \mathrm{MoO}_{3} / \mathrm{Ag}$ & $\begin{array}{l}2.0 \times 10^{-7} \\
(@-5 \mathrm{~V})\end{array}$ & $\begin{array}{l}38.0 \\
\text { (@ 730 nm, -8 V) }\end{array}$ & $\begin{array}{l}6.9 \times 10^{10} \\
(@ \sim 730 \mathrm{~nm},-2 \mathrm{~V})\end{array}$ & $\begin{array}{l}1.5 \mathrm{M} \\
(@-2 \mathrm{~V})\end{array}$ & 10 \\
\hline Chlorinated & $\mathrm{ITO} / \mathrm{ZnO} / \mathrm{ATL} / \mathrm{MoO}_{3} / \mathrm{Ag}$ & $\begin{array}{l}1.7 \times 10^{-10} \\
(@-2 \mathrm{~V})\end{array}$ & $\begin{array}{l}69.1 \\
\text { (@ } 610 \mathrm{~nm},-2 \mathrm{~V} \text { ) }\end{array}$ & $\begin{array}{l}9.6 \times 10^{12} \\
(@ 610 \mathrm{~nm},-2 \mathrm{~V})\end{array}$ & $\begin{array}{l}\text { 110k } \\
(@-5 \mathrm{~V})\end{array}$ & 11 \\
\hline Chlorinated & $\mathrm{ITO} / \mathrm{ZnO} / \mathrm{ATL} / \mathrm{MoO}_{3} / \mathrm{Ag}$ & $\begin{array}{l}2.0 \times 10^{-7} \\
(@-2 \mathrm{~V})\end{array}$ & $\begin{array}{l}10.0 \\
(@ 950 \mathrm{~nm},-2 \mathrm{~V})\end{array}$ & $\begin{array}{l}1 \times 10^{11} \\
(@ 1050 \mathrm{~nm},-2 \mathrm{~V})\end{array}$ & $\begin{array}{l}\sim 1 \mathrm{M} \\
(@-2 \mathrm{~V})\end{array}$ & 12 \\
\hline Chlorinated & $\begin{array}{l}\text { ITO/PEDDOT:PSS/XP2/ATL/LiF } \\
\text { /Al }\end{array}$ & $\begin{array}{l}5.81 \times 10^{-9} \\
(@-0.1 \mathrm{~V})\end{array}$ & $\begin{array}{l}77.5 \\
\text { (@ } 800 \mathrm{~nm},-0.1 \mathrm{~V})\end{array}$ & $\begin{array}{l}1.16 \times 10^{13} \\
(@ 850 \mathrm{~nm},-0.1 \mathrm{~V})\end{array}$ & $\begin{array}{l}\sim 50 \mathrm{k} \\
(@-0.1 \mathrm{~V})\end{array}$ & 13 \\
\hline Chlorinated & $\begin{array}{l}\text { ITO/PCPDTK-Ph/X-IFTPA/ATL/ } \\
\text { LiF/Al }\end{array}$ & $\begin{array}{l}1.1 \times 10^{-8} \\
(@-0.1 \mathrm{~V})\end{array}$ & $\begin{array}{l}49.1 \\
\text { (@ } 1060 \mathrm{~nm},-2 \mathrm{~V})\end{array}$ & $\begin{array}{l}9.86 \times 10^{11} \\
\text { (@1060 nm, -2 V) }\end{array}$ & $\begin{array}{l}\sim 1 \mathrm{M} \\
(@-0.1 \mathrm{~V})\end{array}$ & 14 \\
\hline Chlorinated & $\begin{array}{l}\text { ITO/PEDOT:PSS/ATL/Phen-DPO } \\
\text { /Ag }\end{array}$ & $\begin{array}{l}8.3 \times 10^{-5} \\
(@-2 \mathrm{~V})\end{array}$ & $\begin{array}{l}69.7 \\
\text { (@890 nm, 0 V) }\end{array}$ & $\begin{array}{l}9 \times 10^{11} \\
\text { (@915 nm, 0 V) }\end{array}$ & $\begin{array}{l}\sim 10 \mathrm{k} \\
(@-2 \mathrm{~V})\end{array}$ & 15 \\
\hline Chlorinated & $\mathrm{ITO} / \mathrm{ZnO} / \mathrm{PEI} / \mathrm{ATL} / \mathrm{MoO}_{3} / \mathrm{Ag}$ & $4.0 \times 10^{-8}$ & 24.1 & $4.0 \times 10^{10}$ & $300 \mathrm{k}$ & 16 \\
\hline
\end{tabular}




\begin{tabular}{|l|l|l|l|l|l|l|}
\hline & & $(@ 0 \mathrm{~V})$ & $(@ 1050 \mathrm{~nm},-2 \mathrm{~V})$ & $(@ 1050 \mathrm{~nm}, 0 \mathrm{~V})$ & $(@ 0 \mathrm{~V})$ & \\
\hline Chlorinated & $\mathrm{ITO} / \mathrm{ZnO} / \mathrm{ATL} / \mathrm{P} 3 \mathrm{HT} / \mathrm{Ag}$ & $9.0 \times 10^{-5}$ & 47.4 & $6.59 \times 10^{10}$ & $864 \mathrm{k}$ & 17 \\
& $(@-2 \mathrm{~V})$ & $(@ 560 \mathrm{~nm},-2 \mathrm{~V})$ & $(@ 560 \mathrm{~nm},-2 \mathrm{~V})$ & $(@-2 \mathrm{~V})$ & \\
\hline Chlorinated & $\mathrm{Si} / \mathrm{SiO} / / \mathrm{TiN} / \mathrm{CuSCN} / \mathrm{PCBM} / \mathrm{C} 70 /^{\mathrm{TAPC} / \mathrm{MoO}_{3} / \mathrm{Ag}}$ & $6.0 \times 10^{-10}$ & 15 & $7.15 \times 10^{12}$ & $400 \mathrm{k}$ & 18 \\
& $(@-0.5 \mathrm{~V})$ & $(@ 500 \mathrm{~nm},-1 \mathrm{~V})$ & $(@ 500 \mathrm{~nm},-1 \mathrm{~V})$ & $(@-0.5 \mathrm{~V})$ & \\
\hline
\end{tabular}

\section{The noise spectrum measurement}

The noise spectrum of each sample was measured by the Keysight 35670A dynamic signal analyzer inside Faraday cage.

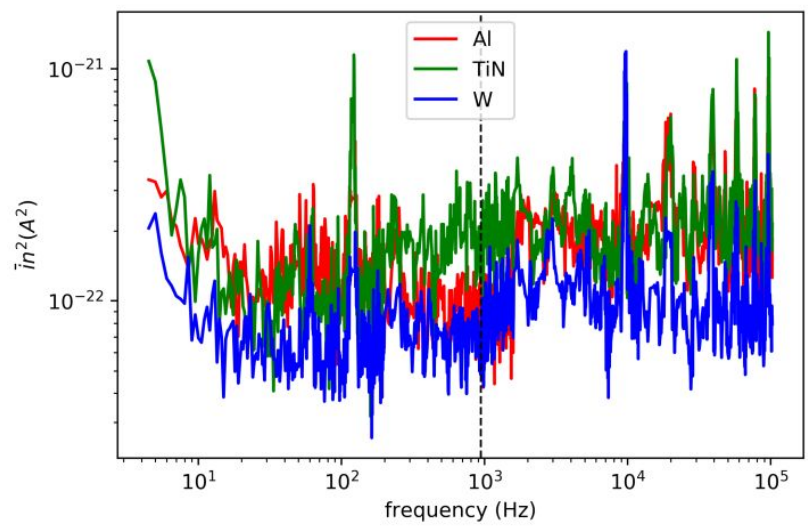

Figure S10. Noise spectrum measurement for OPD with different bottom electrode. 


\section{Reference}

${ }^{1}$ ACS Photonics, 2017, 4, 3, 703-709.

${ }^{2}$ Adv. Electron. Mater., 2017, 1700345.

${ }^{3}$ Adv. Mater. Technol., 2018, 1800158.

${ }^{4}$ Adv. Mater. Technol., 2018, 1800104.

${ }^{5}$ ACS Appl. Mater. Interfaces, 2018, 10, 15, 12937-12946.

${ }^{6}$ IEEE Transactions on Electron Devices, 2018, 65, 4, 1516-1522.

${ }^{7}$ Organic Electronics, 2018, 54, 21-26.

${ }^{8}$ Appl. Phys. Lett., 2020, 117, 093302.

${ }^{9}$ Org. Electron., 2020, 81, 105666.

${ }^{10}$ Adv. Mater., 2020, 1908258.

${ }^{11}$ ACS Appl. Mater. Interfaces, 2020, 12, 43, 48836-48844.

12 J. Mater. Chem. C, 2020, 8, 10098-10103.

13 ACS Appl. Mater. Interfaces, 2020, 12, 40, 45092-45100.

${ }^{14}$ Sci. China Mater. 2021. DOI: org/10.1007/s40843-020-1639-x.

15 J. Mater. Chem. C, 2021, 9, 2375-2380.

${ }^{16}$ Adv. Mater., 2021, 2100582.

${ }^{17}$ Adv. Electron. Mater., 2021, 7, 2000811.

${ }^{18}$ Organic Electronics 67 (2019) 1-9. 